\title{
The influence of facet joint orientation in the lumbar spine segment on the intervertebral disc bulge
}

\author{
KLAUDIA SZKODA-POLISZUK*, MAŁGORZATA ŻAK \\ Department of Mechanics, Materials and Biomedical Engineering, Faculty of Mechanical Engineering, \\ Wrocław University of Science and Technology, Wrocław, Poland.
}

\begin{abstract}
Purpose: Due to the growing percentage of degenerative spinal diseases among the population, it is extremely important to assess how the orientation of the articular facets affects the changes in the intervertebral disc. Therefore, the aim of this study was to assess the effect of the orientation of the articular facets on the changes occurring in individual layers of the annulus fibrosus of the intervertebral disc under the influence of the load causing extension. Methods: Numerical simulations were performed for five configurations of the functional spinal units: physiological, with moderate tropism, severe tropism, and segments in which non-physiological orientation was modelled of both processes with different ranges. Results: It can be concluded that severe tropism causes more significant changes in intervertebral disc bulging on the physiological side of the orientation of the articular facets. Furthermore, the value of stress on articular processes increases tenfold on the side of severe tropism compared to the physiological facet joint orientation. Conclusion: Facet joint orientation plays an important role in the transfer of loads by the spine and the posterior column provides important support for the spine during extension.
\end{abstract}

Key words: finite element method, lumbar spine segment, facet tropism, articular facet orientation, bulging intervertebral disc

\section{Introduction}

The spinal column, composed of alternating vertebrae and intervertebral discs (IVDs), provides appropriate mobility to the human body and is responsible for transfer of loads along the spine axis. Transfer of loads is possible thanks to the three-joint-complex (the "articular triad") consisting of the IVD and two symmetrical facet (zygapophyseal) joints, which connects adjacent vertebrae. The method of support of spinal motion segments - functional spinal units (FSU) - has a significant effect on the kinematics of the spine and the mechanical properties of the IVD [20]. With respect to the location of the points of support, the spine structures can be divided into the anterior and posterior columns. A very important role in the transfer of loads and reduction of excessive mobility of the spine is played by the articular processes of individual FSUs, which, together with vertebral arches, form the posterior column.

Depending on the spine segment (cervical, thoracic, or lumbar), the articular processes of the vertebrae have variable articular facet orientation, i.e., the inclination angle of the articular facets varies depending on the level of the vertebrae in the spinal column. However, within the vertebra itself they are symmetrical to each other (i.e., relative to the sagittal plane). The facet joint orientation is determined as the angle between the sagittal plane and the plane passing through the middle of the facet joint. The inclination angle of the articular facets ranges from $90^{\circ}$ to $110^{\circ}$ in the thoracic segment and from $30^{\circ}$ to $40^{\circ}$ in the lumbar segment [15], [18]. However, there may be some anomalies in the articular facet orientation in the form of an asymmetry of the inclination angle of the ar-

\footnotetext{
* Corresponding author: Klaudia Szkoda-Poliszuk, Department of Mechanics, Materials and Biomedical Engineering, Faculty of Mechanical Engineering, Wrocław University of Science and Technology, ul. Łukasiewicza 7/9, 50-371 Wrocław, Poland. E-mail: klaudia.szkoda-poliszuk@pwr.edu.pl

Received: November 11th, 2020

Accepted for publication: December 9th, 2020
} 
ticular facets between the right and left sides of the vertebra. Although it is believed to be a natural feature of the vertebrae in the thoracic segment, in the case of the lumbar segment, a large asymmetry may cause an abnormal load transfer, which may eventually lead to degenerative changes in the structures of the spine [7], [8], [15], [16]. An asymmetry between the inclination angle of the articular facets within the FSU is referred to as facet tropism [18].

Determining the absolute difference between the right and left sides as well as the distance between articular facets allows for the definition of tropism according to a four-degree grading system of facet joint osteoarthritis (OA) [7], [15]. The first degree is the correct orientation (the difference amounts to between $1^{\circ}$ and $2^{\circ}$, while the distance between the facets is more than $2 \mathrm{~mm}$ ). The second degree is mild tropism (the difference amounts to between $1^{\circ}$ and $2^{\circ}$, while the distance between the facets is more than $2 \mathrm{~mm}$ ). The largest changes in the geometry of the articular processes are observed at moderate tropism (a difference of between $7^{\circ}$ and $15^{\circ}$ ) and severe tropism (a difference greater than $15^{\circ}$ ). At the same time, when moderate tropism occurs, the distance between the articular facets decreases to less than $1 \mathrm{~mm}$; in severe tropism, this distance is practically negligible and the articular facets connect bone to bone [26].

The presence of tropism is generally considered to be an inherited trait, however, sometimes an asymmetry between articular processes can be caused by a scoliotic deformity of the spine [7], [15]. Tropism can be a dangerous phenomenon that, according to numerous studies [5], [19], may lead to degenerative changes in the IVD structures. Do et al. [4] showed a significant correlation between severe tropism $\left(\geq 11^{\circ}\right)$ and increased bulging of the IVD. According to Farfan et al. [5], the orientation of the articular facets towards the frontal plane reduces the torsional (rotational) mechanical strength of the FSU structures, causing damage to the annulus fibrosus (AF). Also, a study by Shirazi-Adl et al. [23] indicates that in the case of facet tropism the IVD becomes damaged only when the rotational movement is combined with bending. On the other hand, some authors point to the lack of correlation between tropism and damage to the IVD structures [8], [10]. However, these reports are rare and it seems highly likely that strong tropism may contribute to the progress of degenerative changes in the lumbar spine [7], [19].

A review of the literature also led the authors to the conclusion that articular processes transfer a significant part of the load, especially during extension, because, as demonstrated by Shirazi-Adl [23], the back- ward bending moment increases the load acting on the articular processes by up to $30 \%$. It can, therefore, be assumed that both compression and forward flexion may have little effect on the changes taking place in the FSU exhibiting facet tropism.

Therefore, the aim of this study was to assess the effect of the orientation of the articular facets on the changes occurring in individual layers of the AF of the IVD under the influence of the load causing extension, which, apart from the torsional load, most clearly exposes the influence of an asymmetry of the articular processes. The changes occurring in successive AF lamellae were analysed on the model of a single FSU (L1-L2) for five different configurations of facet joint orientation. Facet tropism occurs mainly in the lumbar spine [9], most often in the lower levels of the lumbar spine, at the L4-L5 level [10]. However, the upper levels of this spine segment, also those susceptible to tropism, should not be omitted (as the L1-L2 level).

\section{Materials and methods}

\subsection{Development of finite element models}

The FSU of the lumbar spine (L1-L2) was modelled on the basis of spinal dimensions obtained from analysis of computed tomography (CT) images. The CT examination was performed on two men (aged 42 and 45 years) who had no changes in the osteoarticular system of the spine. The CT diagnosis was carried out at the same lateral resolution $(0.37 \mathrm{~mm})$ and thickness of the layer $(0.74 \mathrm{~mm})$. The cross-sectional images (2D) were imported into the InVesalius software, which was used to create 3D models of the L1 and L2 vertebrae in accordance with the methodology set out in the work of Szkoda et al. [24] and SzkodaPoliszuk et al. [25]. The dimensions of both vertebrae were derived from measurements of the 3D models. The obtained values were verified with the vertebral dimensions found in the literature [2], [18]. The geometry of the FSU model of the lumbar spine (L1-L2) was created in the ANSYS Mechanical APDL 18.2 software which was used to perform numerical simulations. The vertebrae and the IVD were described by 10 node tetrahedral solid elements (SOLID187) with 3 degrees of freedom at each node. Each vertebral body was modelled as cancellous bone surrounded by a layer of cortical bone with a thickness in the range of $0.5-0.6 \mathrm{~mm}$ [31]. For both vertebrae, the presence 


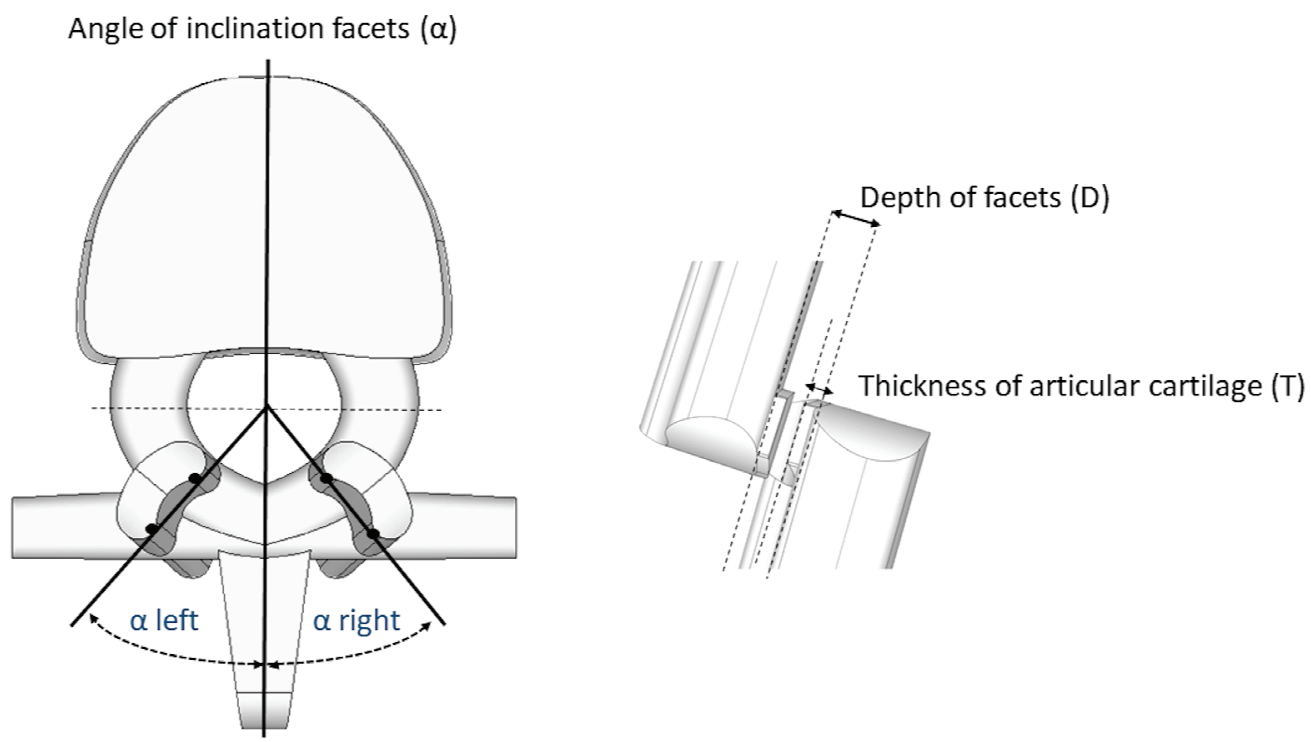

Fig. 1. Measurements of the facet orientation angle. Articular facet orientation is described as the angle of the facet joint in the transverse plane relative to the sagittal plane

Table 1. Configurations of the considered FSU model

\begin{tabular}{|l|c|c|c|c|c|c|}
\hline \multirow{2}{*}{\begin{tabular}{c}
\multirow{2}{*}{$\begin{array}{c}\text { Configurations } \\
\text { of the segment }\end{array}$} \\
\cline { 2 - 7 }
\end{tabular}} & $\alpha\left[^{\circ}\right]$ & $D[\mathrm{~mm}]$ & $T[\mathrm{~mm}]$ & $\alpha\left[{ }^{\circ}\right]$ & $D[\mathrm{~mm}]$ & $T[\mathrm{~mm}]$ \\
\hline Physiological & 35 & 2.50 & 0.65 & 35 & 2.50 & 0.65 \\
\hline Moderate tropism & 35 & 2.50 & 0.65 & 45 & 1.00 & 0.20 \\
\hline Severe tropism & 35 & 2.50 & 0.65 & 55 & 0.40 & 0.05 \\
\hline Non-physiological orientation (moderate) & 45 & 1.00 & 0.20 & 45 & 1.00 & 0.20 \\
\hline Non-physiological orientation (severe) & 55 & 0.40 & 0.05 & 55 & 0.40 & 0.05 \\
\hline
\end{tabular}

$\alpha$ - angle of the facet joint orientation, $D$ - depth of facet, $T$ - thickness of the articular cartilage.

of the superior and inferior endplates was considered, the thickness of which was assumed as $10 \%$ of the vertebral body height [14]. Articular cartilage was modelled on articular processes while taking into account the parameters describing cartilage thickness $(T)$, distance between the processes $(D)$, and the inclination angle $(\alpha)$ of the articular facets (Fig. 1). The thickness of the cartilage and the distance between the processes were determined using the criteria of facet joint OA [7], [15]. The values of the parameters $D, T$, and $\alpha$ for the five analysed configurations are presented in Table 1. All four main types of ligaments found in the spine - anterior longitudinal ligaments (ALL), posterior longitudinal ligaments (PLL), intertransverse ligaments (ITL), and interspinous ligaments (ISL) - were modelled as 2-node link element type LINK10. In the lumbar spine, intertransverse ligament (ITL) is an example of a ligament that is not functionally significant (often not exist at L5-S1 level). However the significant length of the strength arm on which ITL acts, provides it with a significant mechanical advantage over other short ligaments. In contrast, ligamentum flavum (FL) and supraspinous ligament (SSL) although affecting the stability of the spine, can be omitted during biomechanical analysis carried out on a single FSU. This item permits only uniaxial tension with 2 degrees of freedom at each node.

\subsection{Material properties}

The bone tissue of the vertebrae and endplates as well as facet joint cartilage were described by isotropic, linear-elastic material properties [9], [27]-[29] - Table 2. The structure of the AF surrounding the nucleus pulposus (NP) was modelled in the IVD. The AF was modelled as a matrix strengthened with six cylindrically arranged layers of collagen fibres, which were radially located between successive AFs (lamellae). The AF matrix was described by isotropic, linear-elastic material properties [9], [27], [28]. Layers of fibres were 
Table 2. The material properties of the FSU components

\begin{tabular}{|c|c|c|c|c|}
\hline & $\begin{array}{c}\text { Young's modulus } \\
{[\mathrm{MPa}]}\end{array}$ & $\begin{array}{c}\text { Poisson's ratio } \\
{[-]}\end{array}$ & $\begin{array}{c}\text { Cross-section } \\
{\left[\mathrm{mm}^{2}\right]}\end{array}$ & References \\
\hline \multicolumn{5}{|c|}{ SOLID component name } \\
\hline Cortical bone & 12000 & 0.3 & & {$[28],[29]$} \\
\hline Cancellous bone & 100 & 0.2 & & [28], [29] \\
\hline Posterior bone (Neural arch) & 3500 & 0.25 & & {$[9],[27],[28]$} \\
\hline Cartilaginous endplate & 25 & 0.1 & & [9], [27] \\
\hline Nucleus pulposus & 1 & 0.499 & & [9], [13], [27], [29] \\
\hline Annulus ground substance & 4.2 & 0.45 & & [9], [13], [27], [29] \\
\hline Articular cartilage & 33 & 0.3 & & {$[28]$} \\
\hline \multicolumn{5}{|c|}{ LINK component name } \\
\hline $\begin{array}{l}\text { Annulus fibre: } \\
\text { layer } 1 \\
\text { layer } 2 \\
\text { layer } 3 \\
\text { layer } 4 \\
\text { layer } 5 \\
\text { layer } 6 \\
\end{array}$ & $\begin{array}{l}550 \\
495 \\
440 \\
420 \\
385 \\
360\end{array}$ & 0.45 & 0.02 & [9], [11], [13] \\
\hline Anterior longitudial ligaments & 20 & 0.3 & 64 & \multirow{3}{*}[11]{$,[28],[29]$} \\
\hline Posterior longitudial ligaments & 50 & 0.3 & 20 & \\
\hline $\begin{array}{l}\text { Interspinous ligaments } \\
\text { Intertransverse ligaments }\end{array}$ & 12 & 0.3 & 40 & \\
\hline
\end{tabular}

B

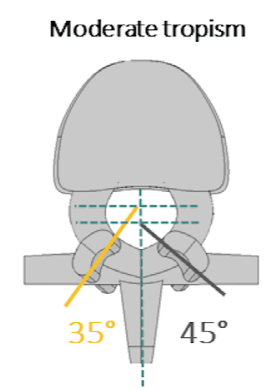

C

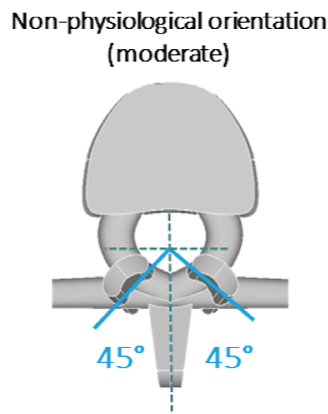

Severe tropism

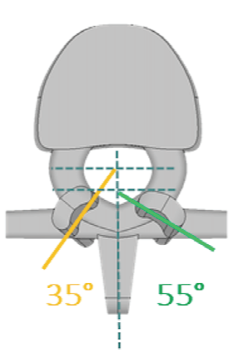

Non-physiological orientation (severe)

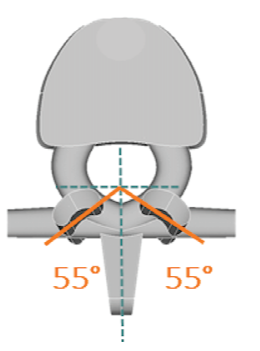

Fig. 2. Configurations of the considered FSU model: a) physiological, b) moderate and severe tropism, c) non-physiological orientation of both processes with different ranges

assigned isotropic, linear material properties with the initial angular orientation of each fibre and theoretically defined cross-sectional area [9], [11], [13]. The fibres were inclined at a $30^{\circ}$ angle to the transverse plane and oriented alternately to the right and left [20]. Ligaments (ALL, PLL, ISL, and ITL) were also described by iso- tropic, linear material properties with theoretically defined cross-sectional area [11], [28], [29]. The articulating facet surfaces were modelled using surface-tosurface contact elements (with a penalty algorithm and a friction coefficient of 0.1 ). The material properties of the individual tissue structures are shown in Table 2. 


\subsection{Boundary and loading conditions}

Numerical simulations were performed for five configurations of the FSU: physiological, with moderate tropism, severe tropism, and segments in which non-physiological orientation was modelled of both processes with different ranges. The considered configurations of the FSU of the lumbar spine are shown in Fig. 2. In view of the fact that tropism causes least interference with the load system of the spine during the action of compressive forces and flexion, the analysis of all configurations was carried out for the load simulating extension, acting with a moment of 10
$\mathrm{Nm}$ [17]. In all cases, the load was applied to the superior surface of the endplate of the vertebral body $\mathrm{L} 1$, and the models were mounted by removing all degrees of freedom of the nodes on the surface of the inferior endplate of the vertebral body L2.

\section{Results}

The mechanical parameters calculated by means of numerical simulations made it possible to specify changes in the IVD. An analysis of the distribution of displacements allowed to determine bulging $\mathrm{B}_{1}$ of indi-

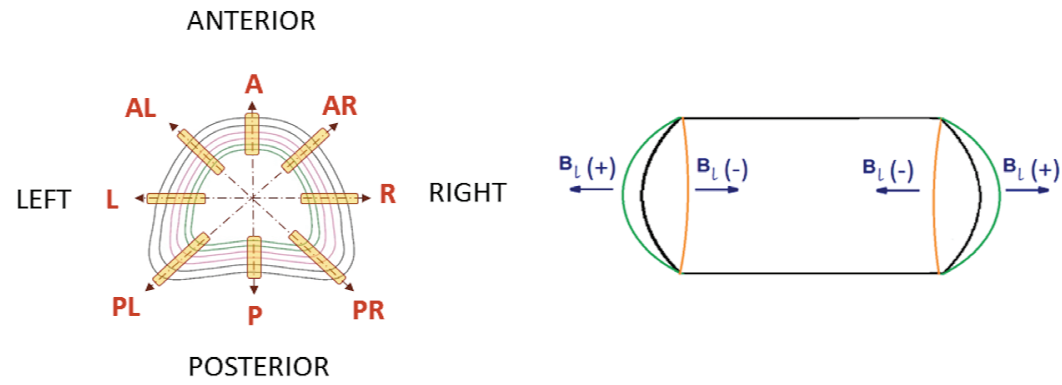

Fig. 3. Analysed regions of IVD bulging and the direction of bulging $\mathrm{B}_{1}$, where: $(+)$ means bulging out of the IVD and (-) means bulging into the IVD

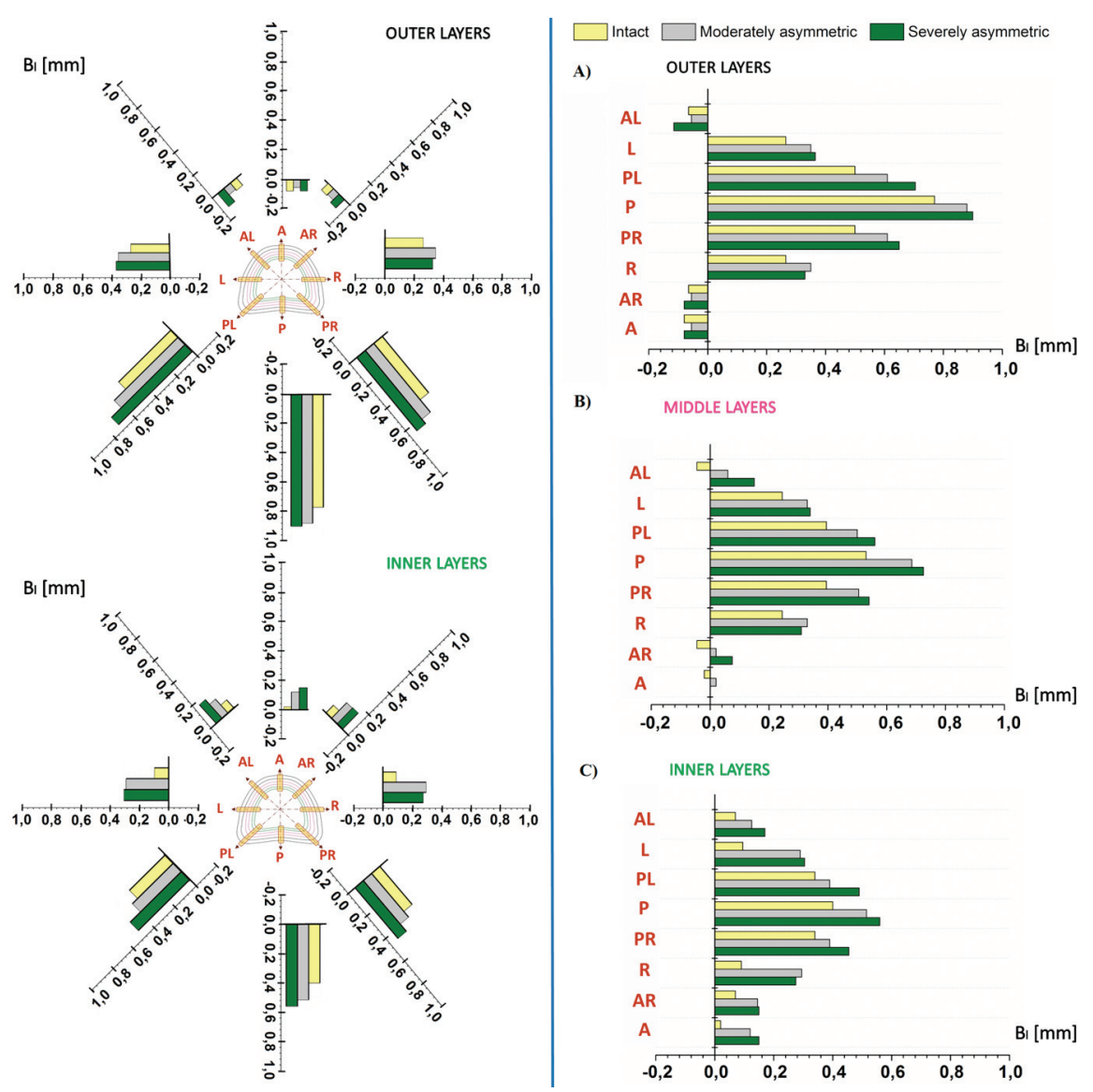

Fig. 4. Bulging $B_{1}$ of individual $A F$ layers (outer $-A$, middle $-B$, inner $-C$ ) in the case of facet tropism 
vidual groups of the AF layers. Six modelled layers of collagen fibres were divided into three groups: outer, middle, and inner. Bulging of these layers was determined in eight of its regions (Fig. 3): in the layers of the anterior $(\mathrm{A})$ and $(\mathrm{P})$ posterior parts relative to the sagittal plane, in the lateral layers $(\mathrm{R}$ - right part, $\mathrm{L}$ - left part) relative to the frontal plane, and also in the regions of the anterior part relative to the plane inclined at an angle of $45^{\circ}$ (AR) and $315^{\circ}(\mathrm{AL})$ and the posterior part relative to the plane inclined at an angle of $135^{\circ}$ (PR) and $225^{\circ}$ (PL).

Depending on the bulging direction of individual layers of the AF, their values were presented as positive (layer bulged out of the IVD) or negative (layer bulged into the IVD). Bulging $\mathrm{B}_{1}$ was determined as differences between the position of the layer before loading (relative to the sagittal plane for the regions A and $\mathrm{P}$, the frontal plane for the regions $\mathrm{R}$ and $\mathrm{L}$, and the plane inclined at the analysed angle for the regions AR, PR, $\mathrm{AL}$, and $\mathrm{PL}$ ), and the position of the layer after loading relative to the same plane. All of the analysed parameters were determined for the five considered configurations of the FSU of the lumbar spine during the application of the load simulating extension.
The analysis showed that the load simulating extension changes the height of the IVD and causes it to bulge outward or inward the IVD depending on the position of a given layer. It was observed that for all five considered configurations of the FSU the bulging of subsequent AF layers decreased with the approach to the NP (Figs. 4, 5). At the same time, it was noted that in the case of the physiological FSU, both outer and middle layers in the anterior part of the IVD bulge inward (in other configurations, external layers only). This is evidenced by the negative values of IVD bulging (values for the outer layers $\mathrm{AL}, \mathrm{A}$, and $\mathrm{AR}$ are, respectively, $-0.065 \mathrm{~mm},-0.080 \mathrm{~mm}$, and $-0.065 \mathrm{~mm}$; values for the middle layer are, respectively, $-0.045 \mathrm{~mm}$, $-0.020 \mathrm{~mm}$, and $-0.045 \mathrm{~mm}$ ). In addition, the observed bulging of the IVD was higher in the posterior part for all layers (PL, P, PR) compared to the anterior and lateral parts (AL, A, AR, R, and L).

\subsection{Changes in the case of facet tropism}

The analysis of the obtained results showed that facet tropism causes a significant change in the system

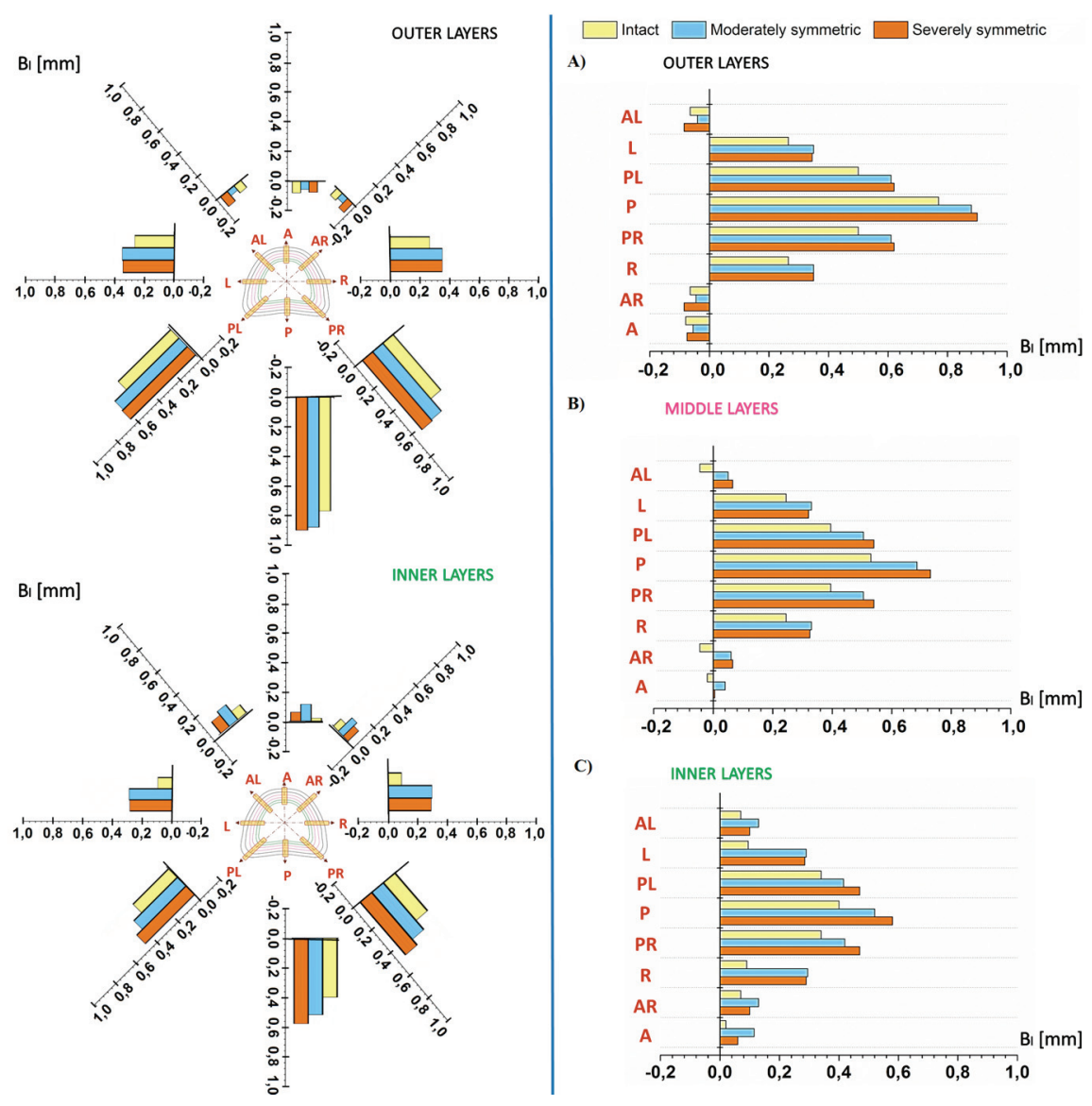

Fig. 5. Bulging $B_{1}$ of individual AF layers (outer $-A$, middle $-B$, inner $-C$ ) in the case of non-physiological orientation of both articular processes 
of load transfer through the IVD structures. In particular, higher bulging of IVD was observed, especially in its posterior part compared to the physiological FSU. The largest changes in IVD bulging occur in the outer layers (Fig. 4). In the posterior part of the IVD (PR, P, and PL) in the case of a physiological FSU, the bulging of the outer layers amounts to, respectively, $0.50 \mathrm{~mm}, 0.70 \mathrm{~mm}$, and $0.50 \mathrm{~mm}$. In the case of moderate tropism in the same regions, these values amount to $0.61 \mathrm{~mm}, 0.88 \mathrm{~mm}$, and $0.61 \mathrm{~mm}$, whereas in severe tropism they amount to $0.65 \mathrm{~mm}$, $0.90 \mathrm{~mm}$, and $0.71 \mathrm{~mm}$. It can, therefore, be also concluded that severe tropism not only increases the bulging values compared to the other two cases, but also causes an asymmetry in the transfer of loads by the FSU, which is not observed in the case of moderate tropism. In the case of severe tropism, the IVD bulging values are higher for all layers on the side of the physiological orientation of the articular facets (left side of the vertebra) than on the right side of the vertebra, where the angle of inclination of the articular facets is greater (Fig. 2). This relationship occurs for both anterior (AR, AL) and posterior (PR, PL) IVD regions as well as for lateral regions $(\mathrm{R}, \mathrm{L})$. On the side of the physiological orientation of the articular facets, the bulging of the outer layer amounts to $-0.12 \mathrm{~mm}$ for the anterior region, $0.37 \mathrm{~mm}$ for the lateral region, and $0.71 \mathrm{~mm}$ for the posterior region. On the right side of the vertebra, where tropism occurs, the same values amount to, respectively, $-0.08 \mathrm{~mm}, 0.33 \mathrm{~mm}$, and $0.65 \mathrm{~mm}$.

\subsection{Changes in the case of non-physiological orientation of both articular facets}

Angular values were chosen analogously to the configurations in which moderate and severe tropism were modelled (Fig. 2). Analysis of the obtained results showed that non-physiological orientation of the articular facets on both sides of the vertebra also contributes to increased bulging of the IVD, especially in its posterior part, compared to the physiological FSU (Fig. 5). The largest changes in IVD bulging occur in the outer layers. In the posterior part of the IVD (PR, $\mathrm{P}$, and PL) in the case of a physiological FSU, the bulging of the outer layers amounts to, respectively, $0.50 \mathrm{~mm}, 0.70 \mathrm{~mm}$, and $0.50 \mathrm{~mm}$. In the case of orientation of the articular facets equal to $45^{\circ}$ in the same regions, these values amount to $0.61 \mathrm{~mm}$, $0.88 \mathrm{~mm}$, and $0.61 \mathrm{~mm}$, while in the case of orientation equal to $55^{\circ}$ they amount to $0.62 \mathrm{~mm}, 0.90 \mathrm{~mm}$, and $0.62 \mathrm{~mm}$. It follows that the obtained values for all layers (outer, middle, and inner) are close to the values obtained for both configurations in which facet tropism occurred. Both for $45^{\circ}$ and $55^{\circ}$ orientations, there is a symmetry in the transfer of loads by the FSU, which is not observed in the case of severe tropism.

\section{Discussion}

Due to the growing percentage of degenerative spinal diseases among the population, it is extremely important to assess how the orientation of the articular facets affects the changes in individual layers of the AF of the IVD caused by loading. According to the studies presented in the literature, tropism can lead to degenerative changes in the IVD structures [4], [19], which very often originate at the border of the $\mathrm{AF}$ and the NP [1], [30]. It is, therefore, important to understand the course of changes occurring in the entire volume of the IVD (both in outer and inner AF lamellae). The conducted experimental studies are very often based on an analysis of global changes in the geometrical or mechanical parameters of the IVD, using for this purpose X-ray imaging, magnetic resonance imaging, or computed tomography. However, these techniques do not allow to specify individual structures (in this case, of the AFs) and thus make it impossible to observe the mechanism of the changes taking place. Consequently, the analysis focuses primarily on changes within the volume of the IVD or its chosen cross-section. Therefore, this work uses the finite element method because it allows to describe qualitative and quantitative changes taking place in individual AF layers.

An analysis of the obtained results showed that, under the influence of the load simulating extension, in each of the considered configurations, almost all layers of the AF bulged outward the IVD. An exception are the outer layers of the AF in the anterior region of the IVD, where there is bulging inward the IVD (and in the case of the physiological FSU, this also applies to the middle layers). The changes taking place in the IVD result from the fact that the acting load (extension) compresses the NP in the posterior region of the IVD (where intra-disc pressure also increases) and pushes it towards the AF lamellae of the anterior region of the IVD, which bulge out [20]. This is also accompanied by a change in the IVD height and a change in the angle of inclination of collagen fibres relative to the surfaces of the vertebral bodies, 
which increases the bulging effect. In addition, extension causes minor stretching of collagen fibres in the anterior part, which changes the value of bulging from positive to negative towards the inside of the IVD). The bulging of the AF layers inward the IVD is dangerous because it can lead to delamination of the AF lamellae, and consequently to degenerative changes of the IVD.

As shown in earlier studies [25], changes in the spinal load transfer system resulting from disorders in the posterior column (articular processes) lead to changes in the anterior column, i.e., a decrease in the height of the IVD and its strong bulging. It can, therefore, be assumed that a strong asymmetry in the orientation of articular facets disturbs the distribution of displacements and strains in the anterior spinal column, as evidenced by the asymmetry concerning the bulging of the IVD (higher values on the physiological side of the IVD). Uneven bulging of the IVD may contribute to the degeneration and damage of the structures of the AF lamellae, especially during extension combined with rotational movement. Rotation within a given FSU causes additional stretching of collagen fibres in the direction of the rotation, which can lead to damage. It is also noteworthy that the values obtained for the physiological FSU presented in this study as well as in previous analyses [25] are close to the bulging values obtained, among others, by Denoziere et al. [3] and Heuer et al. [6]. For presented study, average bulging obtained for anterior region of the IVD amount $0.30 \mathrm{~mm}$ and $0.60 \mathrm{~mm}$ for posterior region of IVD. Previous analyses [25] shown that average bulging obtained for anterior region of the IVD amount $0.40 \mathrm{~mm}$ and $0.60 \mathrm{~mm}$ for posterior region of IVD.

The observed changes within the IVD, and particularly changes in the AFs, are some of the results of the pathological orientation of the articular processes. Also, the articular processes themselves are subjected to negative changes resulting from an asymmetrical or nonphysiological position. An analysis of the values of the contact stress occurring on the articular facets shows how significant changes take place on these cooperating facets in the case of their incorrect orientation (Fig. 6). The obtained results showed that, in the case of the physiological segment, the stress values on both sides amount to about 2.7 $\mathrm{MPa}$. The greatest changes are observed for severe tropism, where on the side of its presence the stress increases tenfold to $27 \mathrm{MPa}$. In addition, almost twice higher stress (about $5.5 \mathrm{MPa}$ ) is observed in the case of non-physiological orientation of the articular facets, whose inclination angle is $45^{\circ}$, and almost six times higher stress (17 MPa) for the inclination angle equal to $55^{\circ}$. Moderate tropism causes only a slight increase in stress (by about $2 \mathrm{MPa}$ ) on the side of its presence compared to the physiological orientation of the articular facets.

Such high increase in stress on articular processes also indicates their overloading, which in the long term will lead to degenerative changes of these elements. This, in turn, will increase the pathological changes in the IVD itself.

The presented research results possibly indicate that the presence of tropism may contribute to degen-
RIGHT

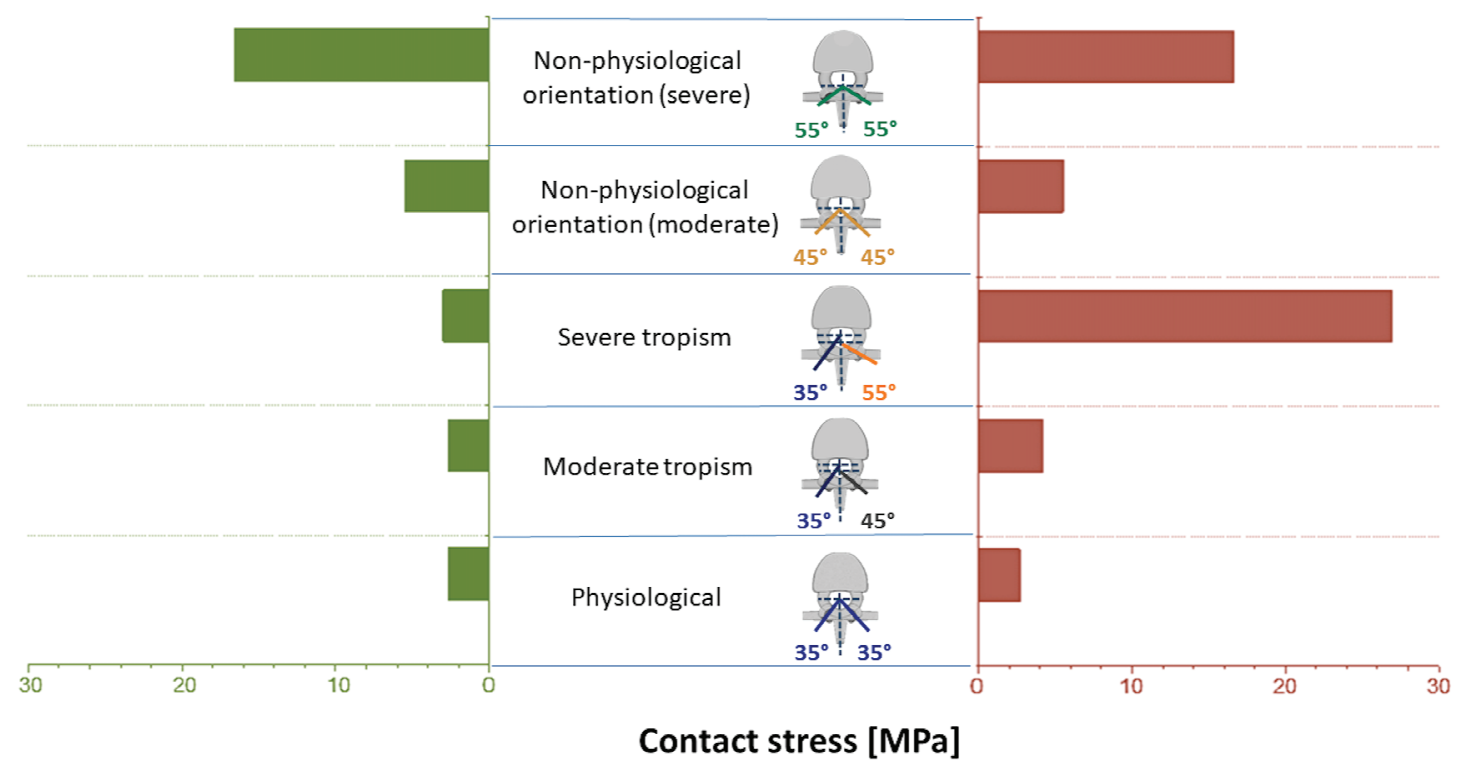

Fig. 6. Stress occurring in the facet joints on the articular facet surfaces on the left and right sides of the FSU 
erative changes both in the region of the IVD as well as the region of facet joints, as evidenced by excessively asymmetrical bulging of the IVD or excessive stress on the articular facets on the side of tropism. Similar dependencies were presented in the studies by Rong et al. [21] and Liu et al. [12], who demonstrated in numerical and in vivo studies the increased values of the force acting on the articular facets in the segments taking the occurrence of tropism into account. At the same time, Liu et al. [12] observed that the greater the inclination angle of the articular facets, the higher the value of the force acting on these facets. This is an important cognitive element in biomechanics of the spine because, as shown by the available research work, the presence of tropism may play a key role in making treatment decisions and predicting the progress of osteoarthritis. Therefore, Samartzis et al. [22] suggest that diagnosis and imaging of vertebral structures should consider the orientation of the articular facets and the presence of tropism, especially in the lumbar spine.

The presented solution of the problem was in some aspects simplified, which is of course a limitation of the conducted analysis, but it makes it possible to demonstrate the effect of the orientation of the articular facets on the changes occurring in the individual layers of the AF of the IVD. Necessary simplifications and assumptions concerned the geometry of FSU and applied loads. Due to the fact that the IVD was the object of the analysis, its structure was precisely mapped, modelling in it collagen fibres as linear elements with various material properties, which is also proposed by authors of other numerical studies [9], [13]. This allowed for the representation of the physiological structure of the IVD, in which the outer lamellae of the AF have higher mechanical properties than the inner lamellae. The introduced simplifications concerned the properties of the bone tissue, the NP, and the AF matrix, because they were modelled as structures with the properties of an isotropic material. This also allowed to optimise the time of the conducted numerical simulations. It should be emphasized that the considered model in terms of assumed material properties of the different tissues is extremely complex. Therefore, a more accurate geometry of the FSU model of the lumbar spine could not contribute much to the analysis of the issue.

\section{Conclusions}

The obtained results for the analysed cases led to the following conclusions:
- facet joint orientation plays an important role in the transfer of loads by the spine and the posterior column provides important support for the spine during extension,

- the largest change in IVD bulging occur in the case of inner layers of the AF,

- facet tropism or the presence of their non-physiological orientation on both sides causes significant bulging of the IVD (especially in its posterior part) compared to the IVD in the physiological system,

- severe tropism causes more significant changes in IVD bulging on the physiological side of the orientation of the articular facets,

- the value of stress on articular processes increases tenfold on the side of severe tropism compared to the physiological facet joint orientation.

\section{Acknowledgements}

Calculations have been carried out using resources provided by Wroclaw Centre for Networking and Supercomputing (http://wcss.pl), grant No. 423

\section{References}

[1] Adams M.A., McNally D.S., Dolan P., Stress distributions inside intervertebral discs. The effects of age and degeneration, J. Bone Joint Surg. Br., 1996, 76 (6), 965-972, DOI: 10.1302/0301-620x78b6.1287.

[2] Berry J.L., Moran J.M., Berg W.S., Steffee A.D., A morphometric study of human lumbar and selected thoracic vertebrae, Spine (Phila, Pa 1976), 1987, 12 (4), 362-367, DOI: 10.1097/00007632-198705000-00010.

[3] DenOzIÈRE G., Ku D.N., Biomechanical comparison between fusion of two vertebrae and implantation of an artificial intervertebral disc, J. Biomech., 2006, 39 (4), 766-775, DOI: 10.1016/j.jbiomech.2004.07.039.

[4] Do D.H., Taghavi C.E., Fong W., Kong M.H., Morishita Y., WANG J.C., The relationship between degree of facet tropism and amount of dynamic disc bulge in lumbar spine of patients symptomatic for low back pain, Eur. Spine J., 2011, 20, 71-78, DOI: 10.1007/s00586-010-1558-8.

[5] FARFAn H.F., Sullivan J.D., The relation of facet orientation to intervertebral disc failure, Can. J. Surg., 1967, 10 (2), 179-185.

[6] Heuer F., SchmidT H., Wilke H.J., The relation between intervertebral disc bulging and annular fiber associated strains for simple and complex loading, J. Biomech., 2008, 41 (5), 1086-1094, DOI: 10.1016/j.jbiomech.2007.11.019.

[7] Kalichman L., Suri P., Guermazi A., Li L., Hunter D.J., Facet orientation and tropism, associations with facet joint osteoarthritis and degenerative spondylolisthesis, Spine, 2009, 34 (16), E579, DOI: 10.1097/BRS.0b013e3181aa2acb.

[8] Karacan I., Aydin T., Sahin Z., Cidem M., Koyuncu H., AKTAS I., Uludag M., Facet angles in lumbar disc herniation, their relation to anthropometric features, Spine, 2004, 29 (10), 1132-1136, DOI: 10.1097/00007632-200405150-00016. 
[9] Kim H.J., TaK Kang K., Chang B.S., LeE CH.K., Kim J.W. et al., Biomechanical analysis of fusion segment rigidity upon stress at both the fusion and adjacent segments - a comparison between unilateral and bilateral pedicle screw fixation, Yonsei Med. J., 2014, 55, 1386-1394, DOI: 10.3349/ymj.2014.55.5.1386.

[10] Kong M.H., He W., Tsai Y.D., Chen N.F., Keorochana G., Do D.H. et al., Relationship of facet tropism with degeneration and stability of functional spinal unit, Yonsei Med. J., 2009, 5, 624-629, DOI: 10.3349/ymj.2009.50.5.624.

[11] Li Q.Y., Kim H.J., Son J., Kang K.T., Chang B.S., Lee C.K., YeOM J.S., Biomechanical analysis of lumbar decompression surgery in relation to degenerative changes in the lumbar spine - validated finite element analysis, Comput. Biol. Med., 2017, 89, 512-519, DOI: 10.1016/j.compbiomed.2017.09.003.

[12] Liu X., Huang Z., Zhou R., Zhu Q., Ji W., Long Y., Wang J., The Effects of Orientation of Lumbar Facet Joints on the Facet Joint Contact Forces. An In Vitro Biomechanical Study, Spine, 2018, 43 (4), E216-E220, DOI: 10.1097/ BRS.0000000000002290.

[13] López E., Elena I., Herrera A., Mateo J., Lobo-Escolar A. et al., Probability of osteoporotic vertebral fractures assessment based on DXA measurements and finite element simulation, Adv. Biosci. Biotechnol., 2014, 5, 527-545, DOI: 10.4236/ abb.2014.56063.

[14] Lotz J.C., Fields A.J., LiebenberG E.C., The role of the vertebral endplate in low back pain, Global Spine J., 2013, 3 (3), 153-164, DOI: 10.1055/s-0033-1347298.

[15] Masharawi Y., Rothschild B., Dar G., Peleg S., Robinson D., BeEn E., HershKovitz I., Facet orientation in the thoracolumbar spine, three-dimensional anatomic and biomechanical analysis, Spine, 2004, 29 (16), 1755-1763, DOI: 10.1097/01.BRS.0000134575.04084.EF.

[16] MichNiK R., ZADOŃ H. et al., The effect of the pelvis position in the sagittal plane on loads in the human musculoskeletal system, Acta Bioeng. Biomech., 2020, 22 (3), 33-42, DOI: 10.37190/ABB-01606-2020-02.

[17] Panjabi M.M., BRAnd R.A., White A.A., Mechanical properties of the human thoracic spine as shown by three-dimensional load-displacement curves, J. Bone Joint Surg. Am., 1976, 58 (5), 642-652, DOI: 10.2106/00004623-197658050-00011.

[18] Panjabi M.M., Oxland T., Takata K., Goel V., Duranceau J., Krag M., Articular facets of the human spine. Quantitative threedimensional anatomy, Spine (Phila, $\mathrm{Pa}$ 1976), 1993, 18 (10), 1298-1310, DOI: 10.1097/ 00007632-199308000-00009.

[19] Pichaisak W., Chotiyarnwong C., Chotiyarnwong P., Face joint orientation and tropism in lumbar degenerative disc disease and spondylolisthesis, J. Med. Assoc. Thai., 2015, 98 (4), 373-379, DOI: 10.1055/s-0034-1376586.
[20] Rohlmann A., Zander T., Schmidt H., Wilke H.J., Bergmann G., Analysis of the influence of disc degeneration on the mechanical behavior of a lumbar motion segment using the finite element method, J. Biomech., 2006, 39 (13), 2484-2490, DOI: 10.1016/j.jbiomech.2005.07.026.

[21] Rong X., LiU Z., Wang B., PAN X., LiU H., Relationship between facet tropism and facet joint degeneration in the sub-axial cervical spine, BMC Musculoskeletal Disorders, 2017, 18 (1), 86, DOI: 10.1186/s12891-017-1448-x.

[22] Samartzis D., Cheung J.P., Rajasekaran S., Kawaguchi Y., ACHARYA S., KAWAKAMI M. et al., Critical values of facet joint angulation and tropism in the development of lumbar degenerative spondylolisthesis: an international, large-scale multicenter study by the AO Spine Asia Pacific Research Collaboration Consortium, Global Spine J., 2016, 6, 414-421, DOI: $10.1055 / \mathrm{s}-0035-1564417$.

[23] ShiraZi-Adl A., Drouin G., Load-bearing role of facets in a lumbar segment under sagittal plane loadings, J. Biomech., 1987, 20 (6), 601-613, DOI: 10.1016/0021-9290(87)90281-8.

[24] Szkoda K., Pezowicz C.A., Finite element analysis of fixation system influence on the thoracolumbar spine stability, Appl. Mech. Mater., 2016, 821, 685-692, DOI: 10.4028/ www.scientific.net/AMM.821.685.

[25] Szkoda-Poliszuk K., ŻAK M., Pezowicz C., Finite element analysis of the influence of three-joint spinal complex on the change of the intervertebral disc bulge and height, Int. J. Numer. Meth. Biomed. Engng., 2018, 34 (9), e3107, DOI: 10.1002/cnm.3107.

[26] Weishaupt D., ZANETti M., Boos N. et al., MR imaging and CT in osteoarthritis of the lumbar facet joints, Skeletal Radiol., 1999, 28, 215-219, DOI: 10.1007/s002560050503.

[27] Xu M., YANG J., Lieberman I.H., HAdDAS R., Lumbar spine finite element model for healthy subjects: development and validation, Comput. Methods Biomech. Biomed. Eng., 2017, 20 (1), 1-15, DOI: 10.1080/10255842.2016.1193596.

[28] Zahaf S., HabiB H., Mansouri B., Belarbi A., AZARi Z., The effect of the eccentric loading on the components of the spine, GJRE, 2016, 4 (1), 6-22, DOI: 10.15761/HMO.1000110.

[29] ZAHARI S.N., LATIF M.J.A., RAHIM N.R.A., KADIR M.R.A., KAMARUL T., The effects of physiological biomechanical loading on intradiscal pressure and annulus stress in lumbar spine: a finite element analysis, J. Healthc. Eng., 2017, 9618940, DOI: 10.1155/2017/9618940.

[30] ŻAK M., Effect of support on mechanical properties of the intervertebral disc in long-term compression testing, J. Theor. Appl. Mech., 2014, 52 (3), 677-686.

[31] ZANDER T., ROHLMANN A., BERGMANN G., Influence of different artificial disc kinematics on spine biomechanics, Clin. Biomech., 2009, 24 (2), 135-142, DOI: 10.1016/j.clinbiomech.2008.11.008. 